\title{
A Simpler and More Direct Derivation of System Reliability Using Markov Chain Usage Models
}

\author{
Lan Lin, Yufeng Xue \\ Department of Computer Science \\ Ball State University \\ Robert Bell Building, Room 455 \\ Muncie, IN 47306, USA \\ \{llin4, yxue2\}@bsu.edu
}

\author{
Fengguang Song \\ Department of Computer and Information Science \\ Indiana University-Purdue University Indianapolis \\ 723 W. Michigan St., SL 280 \\ Indianapolis, IN 46202, USA \\ fgsong@cs.iupui.edu
}

\begin{abstract}
Markov chain usage-based statistical testing has been around for more than two decades, and proved sound and effective in providing audit trails of evidence in certifying software-intensive systems. The system end-to-end reliability is derived analytically in closed form, following an arc-based Bayesian model. System reliability is represented by an important statistic called single use reliability, and defined as the probability of a randomly selected use being successful. This paper reviews the analytical derivation of the single use reliability mean, and proposes a simpler, faster, and more direct way to compute the expected value that renders an intuitive explanation. The new derivation is illustrated with two examples.
\end{abstract}

\section{Introduction}

Statistical testing based on a Markov chain usage model, as a rigorous testing method developed by the University of Tennessee Software Quality Research Laboratory (UTK SQRL), has been around for more than two decades $[6,5,10,8,11,14,16,15]$ and successfully applied in a variety of industry and government projects, ranging from medical devices to automotive components to scientific instrumentation, to name a few $[3,2,13]$. The direct benefit statistical testing provides is a quantitative analysis of the system's quality using empirical data that can be used to demonstrate, document, and certify that the system is fit for its intended use.

In statistical testing a Markov chain usage model is first developed. States of the Markov chain represent states of system use (such as "Cruise Control Activated," "Cruise Control Deactivated," or "Cruise Control Resumed" in an automobile cruise control system). Arcs between states rep- resent possible transitions between states of use (such as "Activating Cruise Control" when the driver presses a button on the steering wheel, or "Deactivating Cruise Control" when the driver engages the brake). Each arc has an associated probability of making that particular transition, based on expected usage data in the field. The outgoing arcs from each state have probabilities that sum to one. Test cases can be generated from the model by different sampling options. Pass and fail data are recorded and analyzed for reliability estimation, coverage analysis, or stopping decisions. This form of statistical testing $[6,5,10,8,11,14,16,15]$ supports quantitative certification of software by a statistical protocol. A public domain tool supporting statistical testing (JUMBL: J Usage Model Builder Library developed by UTK SQRL) is freely available $[9,1]$.

This paper reviews the current reliability analysis for statistical testing based on a Markov chain usage model, and specifically how to derive a system end-to-end reliability estimate based on the testing experience given the usage model, as established in $[10,12]$, and proposes a simpler way to compute the expected value of system reliability, not via the system failure probability (or unreliability), but directly. The new derivation results in a simpler closed-form formula with less steps of computation that also renders an intuitive explanation. We illustrate it through two examples and compare the results with those obtained from the old analysis.

The remainder of the paper is organized as follows. Section 2 discusses related work and compares different reliability models that have been used for statistical testing. Section 3 reviews the current analytical derivation of the system reliability. Section 4 presents our new formula with two illustrating examples. Finally Section 5 concludes the paper and points out directions for future work. 


\section{Related Work: Reliability Analysis}

After a sample of test cases are generated from the usage model and executed, results of testing are recorded. A test case is considered successful if all the steps (events) constituting the test case are executed successfully; otherwise, the steps on which a test case fails are recorded, together with the information as to whether testing continues or stops after each failure step. The recorded testing results are empirical data used to estimate the system reliability.

Several reliability models that have been used for statistical testing are presented and compared in $[10,12]$. They are briefly described below:

- The sampling model [7]. Each executed test case is a Bernoulli trial. An estimated reliability is computed at a confidence level, based on the numbers of successful and unsuccessful test cases. Variations among tests are not taken into account, with long and short tests equally treated.

- The failure state model [16]. The testing result is stored as a testing (Markov) chain. It contains states from the usage model that have been visited during testing, and additional failure states for observed failures. The failure states are made absorbing. The upper bound on system reliability is defined as the probability of going from the source to the sink of the testing chain without being absorbed in a failure state. The model takes into account the probability mass of each test case, however, in the absence of failures (no absorbing states) the estimate of reliability is one.

- The Bayesian model [4]. Miller et al. presented a reliability model based on Bayesian theory. It allows for the use of prior (testing) information, and assumes a standard beta distribution for the software failure probability (or "unreliability," that is, the probability of a randomly chosen use failing). An expected value for system reliability and the associated variance are computed from a posterior beta distribution, based on both prior and observed successes and failures.

- The arc-based Bayesian model [10, 12]. The Miller model is applied to individual arcs of a Markov chain usage model. For each arc the expected value of the arc failure probability and the associated variance are computed from a posterior beta distribution. A single use reliability is defined as "the probability of the software executing a randomly selected use without a failure." This model takes into account variations among tests, makes use of prior testing information, yields a reliability estimate based on the amount of testing when testing reveals no failures, and provides a variance estimate.
The arc-based Bayesian approach is appealing because first, it takes into account variations among tests induced by the model structure; second, it provides insight into where lack of testing or unreliability is having a greater impact on the system level end-to-end reliability, as a failure on one arc may not have the same impact as a failure on a different arc; and third, it makes it easier for the tester to estimate the pre-test reliability for a single arc, which contributes to a more accurate estimate of the system reliability. For these reasons the latest version of the JUMBL [1] adopts the arcbased Bayesian model.

\section{Single Use Reliability and Its Derivation}

Using the arc-based Bayesian model one could compute the system end-to-end reliability through the single use reliability estimate, either via analytical derivation [10] or through simulation [12]. Single use reliability is defined as "the probability of a randomly selected use executing correctly relative to a specification of correct behavior $[6,10]$." Since the analytical solution in closed form [10] is both faster and more precise than simulation, it was implemented in the latest JUMBL tool. We summarize the major steps and results of the derivation below.

Let $P=\left[p_{i j}\right]$ be the $n \times n$ transition matrix of a Markov chain usage model. The $(i, j)$-th entry $p_{i j}$ of $P$ is the conditional probability of the next state being state $j$ given the current state being state $i$. State 1 is the source. State $n$ is the sink and the only absorbing state (assuming a reasonable error recovery scheme). Given $P_{n \times n}, Q_{(n-1) \times n}$ denotes the submatrix of $P$ omitting the last row, and $\dot{Q}_{(n-1) \times(n-1)}$ denotes the submatrix of $P$ omitting the last row and the last column. $\dot{Q}$ is the transition matrix of the Markov chain restricted to the transient states.

Let $r_{i, j}$ be a random variable for "transition reliability," that is, the fraction of successful transitions from state $i$ to state $j$. Let $f_{i, j}$ be another random variable for "transition failure probability," that is, the fraction of unsuccessful transitions from state $i$ to state $j$. Notice that $f_{i, j}=1-r_{i, j}$.

With the arc-based Bayesian model [12], each arc (transition) reliability $r_{i, j}$ has a standard beta distribution $B\left(\alpha_{i, j}, \beta_{i, j}\right)$ with two parameters $\alpha_{i, j}$ (for total successes on transitions from state $i$ to state $j$ ) and $\beta_{i, j}$ (for total failures on transitions from state $i$ to state $j$ ), where $\alpha_{i, j}=a_{i, j}+s_{i, j}$ and $\beta_{i, j}=b_{i, j}+f_{i, j}$ with $a_{i, j}, s_{i, j}$, $b_{i, j}, f_{i, j}$ representing prior successes, observed successes, prior failures, and observed failures, respectively, on transitions from state $i$ to state $j$. The prior success and failure counts are estimated from prior testing experience or knowledge obtained from code analysis, design records, previous failure data, etc. In case no prior information is available, $a_{i, j}=b_{i, j}=1$. The observed success and failure counts are collected through testing. Each executed test 
case is mapped to the usage model and each executed step is marked as successful or failing. The observed success and failure counts are summed for each individual arc in the usage model.

From the posterior (beta) distribution $B\left(\alpha_{i, j}, \beta_{i, j}\right)$ for $r_{i, j}$ we may compute the mean of $r_{i, j}$ :

$$
E\left[r_{i, j}\right]=\frac{\alpha_{i, j}}{\alpha_{i, j}+\beta_{i, j}}=\frac{a_{i, j}+s_{i, j}}{a_{i, j}+s_{i, j}+b_{i, j}+f_{i, j}} .
$$

Given $f_{i, j}=1-r_{i, j}$, we can compute the mean of $f_{i, j}$ as $E\left[f_{i, j}\right]=E\left[1-r_{i, j}\right]=1-E\left[r_{i, j}\right]$.

By our assumption state $n$ (the sink) is the only absorbing state of the Markov chain. A test case ends when the sink is first encountered, therefore, we are only interested in transitions from any state other than the sink (any transient state). In the matrices defined below ( $A, B, S, F, R_{1}$, and $\left.F_{1}\right), i$ is any integer from 1 to $n-1$ inclusive, and $j$ is any integer from 1 to $n$ inclusive.

Let $A=\left[a_{i, j}\right]$ and $B=\left[b_{i, j}\right]$ be two matrices of size $(n-1) \times n$ whose entries are prior arc successes and failures, respectively, obtained from prior testing experience. Let $S=\left[s_{i, j}\right]$ and $F=\left[f_{i, j}\right]$ be two matrices of size $(n-1) \times n$ whose entries are observed arc successes and failures, respectively, obtained through testing.

Let $R_{1}=\left[E\left[r_{i, j}\right]\right]$ be an $(n-1) \times n$ matrix whose $(i$, $j$ )-th entry is the expected arc reliability of going from state $i$ to state $j$, and $\dot{R}_{1}$ denote the submatrix of $R_{1}$ omitting the last column.

Similarly we define $F_{1}=\left[E\left[f_{i, j}\right]\right]$ as an $(n-1) \times n$ matrix whose $(i, j)$-th entry is the expected arc failure probability of going from state $i$ to state $j$.

Given two matrices $X$ and $Y$ of the same size (dimension), $X \otimes Y$ denotes the entry-wise (or component-wise) product of $X$ and $Y . X \otimes Y$ has the same size as $X$ and $Y$.

We define two entry-wise products as follows. One is of size $(n-1) \times n: \mathscr{F}_{1}=Q \otimes F_{1}$. The other is a square matrix of order $n-1: \dot{\mathscr{R}}_{1}=\dot{Q} \otimes \dot{R}_{1}$.

Let $I$ be an $(n-1) \times(n-1)$ identity matrix, and $U$ be a column vector of ones of size $n$. It is established in [10] that $F^{*}$ in (1) computes the expected single use failure probability (or single use unreliability) from any starting state.

$$
F^{*}=\left(I-\dot{\mathscr{R}}_{1}\right)^{-1} \mathscr{F}_{1} U
$$

Observe that $F^{*}$ is a column vector of size $n-1$. The $i$-th component of $F^{*}$ is the computed probability of failure (the expected value) for an arbitrary use of the system from a particular usage state, state $i$, to the sink ( $i$ runs from 1 to $n-1$ inclusive; the starting state could be any transient state).

Therefore, the expected single use reliability of the system (starting from the source) is one minus the first component of $F^{*}$ computed by (1).

For an intuitive understanding of (1), consider all the paths in the usage model that originate from state $i$ and have all but the last step successful; the last step on the path is the only failure step. The probability of taking one of such paths gives the failure probability from state $i$, and is computed in three steps. First, it is shown in [10] that $\left(I-\dot{\mathscr{R}}_{1}\right)^{-1}=\dot{\mathscr{R}}_{1}^{0}+\dot{\mathscr{R}}_{1}^{1}+\dot{\mathscr{R}}_{1}^{2}+\ldots$, hence the $(i, j)$ th entry in the inverse matrix computes the probability of successfully moving from state $i$ to state $j$ in any finite and arbitrary number of steps (starting from 0 step). State $j$ must be transient because only the last failure step could lead to the sink. Second, the inverse matrix is multiplied by the single-step failure matrix $\mathscr{F}_{1}$ to give the probability of moving from any transient state to any state in the model with all but the last step successful. Here the last transition is made to either a transient state or the sink. And last, the product is multiplied by the vector of ones of appropriate size to sum up the probabilities of taking paths with a fixed starting state, all successful prior steps before encountering the last failure step, and an arbitrary ending state. The sum is the failure probability from the particular starting state.

A more complex equation is also given in [10] for computing the variance associated with the single use reliability from any starting state, which we are not elaborating here due to the page limits.

To sum up, the following steps are required to compute the system reliability mean as illustrated in [10]:

1. Determine $Q$ and $\dot{Q}$ from the usage model.

2. Determine $A$ and $B$ from prior success and failure counts for each arc in the usage model.

3. Determine $S$ and $F$ from observed success and failure counts for each arc in the usage model.

4. Compute $R_{1}$ and $F_{1}$ from $A, B, S$, and $F$.

5. Compute $\dot{\mathscr{R}}_{1}$ and $\mathscr{F}_{1}$.

6. Compute $F^{*}$ by (1).

7. The expected value of the single use reliability is one minus the first component of $F^{*}$.

\section{A Simpler and More Direct Derivation of the Single Use Reliability Mean}

We think the derivation of the single use reliability mean (expected value) could be simplified, and not through the single use failure probability (or single use unreliability) but directly, as shown below.

\subsection{The New Derivation}

We define another entry-wise product of size $(n-1) \times n$ : $\mathscr{R}_{1}=Q \otimes R_{1}$. Let $W$ be $\mathscr{R}_{1}$ restricted to the last column. $\mathscr{R}_{1}$ is a column vector of size $n-1$.

We define $R^{*}$ as follows:

$R^{*}=\left(I-\dot{\mathscr{R}}_{1}\right)^{-1} W$ 


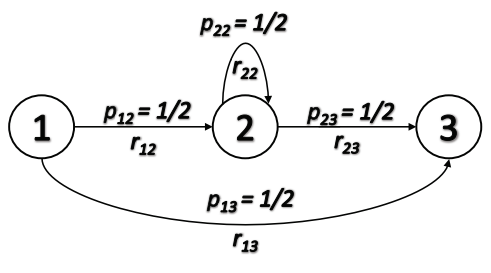

Figure 1. Example 1 of a Markov chain usage model. Arcs are annotated with transitional probabilities and arc reliabilities.

(2) has an intuitive explanation. As explained above for $(1)$, the $(i, j)$-th entry in the inverse matrix computes the probability of successfully moving from the transient state $i$ to the transient state $j$ in any finite and arbitrary number of steps (starting from 0 step). When multiplied by the single-step success matrix $\mathscr{R}_{1}$ restricted to the last column (i.e., $W$ ), the last steps are successful steps leading to the sink, hence $R^{*}$ gives the probability of successfully moving from any transient state to the sink in any finite and arbitrary number of steps (starting from 0 step).

Observe that $R^{*}$ is a column vector of size $n-1$. The $i$-th component of $R^{*}$ is the expected single use reliability starting from state $i$ ( $i$ runs from 1 to $n-1$ inclusive).

Therefore, the expected single use reliability of the system (starting from the source) is the first component of $R^{*}$ computed by (2).

We demonstrate below two examples of computing the single use reliability mean by the formula in [10] (hereafter referred to as Stacy and Poore's formula) as well as by our new formula. For both examples the new formula gets to the same result with fewer steps and simplified calculation.

\subsection{Examples}

Figure 1 illustrates our first example of a Markov chain usage model. The arcs are annotated with transitional probabilities and arc reliabilities.

By Stacy and Poore's formula:

$$
\begin{aligned}
& P=\left[\begin{array}{lll}
0 & \frac{1}{2} & \frac{1}{2} \\
0 & \frac{1}{2} & \frac{1}{2} \\
0 & 0 & 0
\end{array}\right] \quad Q=\left[\begin{array}{lll}
0 & \frac{1}{2} & \frac{1}{2} \\
0 & \frac{1}{2} & \frac{1}{2}
\end{array}\right] \quad \dot{Q}=\left[\begin{array}{ll}
0 & \frac{1}{2} \\
0 & \frac{1}{2}
\end{array}\right] \\
& R_{1}=\left[\begin{array}{lll}
r_{11} & r_{12} & r_{13} \\
r_{21} & r_{22} & r_{23}
\end{array}\right] \quad \dot{R}_{1}=\left[\begin{array}{ll}
r_{11} & r_{12} \\
r_{21} & r_{22}
\end{array}\right] \quad I=\left[\begin{array}{ll}
1 & 0 \\
0 & 1
\end{array}\right] \\
& \dot{\mathscr{R}}_{1}=\dot{Q} \otimes \dot{R}_{1}=\left[\begin{array}{ll}
0 & \frac{r_{12}}{2} \\
0 & \frac{r_{22}}{2}
\end{array}\right] \quad I-\dot{\mathscr{R}}_{1}=\left[\begin{array}{cc}
1 & -\frac{r_{12}}{2} \\
0 & 1-\frac{r_{22}}{2}
\end{array}\right] \\
& \left(I-\dot{\mathscr{R}}_{1}\right)^{-1}=\frac{1}{1-\frac{r_{22}}{2}}\left[\begin{array}{ccc}
1-\frac{r_{22}}{2} & \frac{r_{12}}{2} \\
0 & 1
\end{array}\right]=\left[\begin{array}{ll}
1 & \frac{r_{12}}{1-\frac{r_{22}}{2}} \\
0 & \frac{1}{1-\frac{r_{22}}{2}}
\end{array}\right] \\
& F_{1}=1-R_{1}=\left[\begin{array}{lll}
1-r_{11} & 1-r_{12} & 1-r_{13} \\
1-r_{21} & 1-r_{22} & 1-r_{23}
\end{array}\right]
\end{aligned}
$$

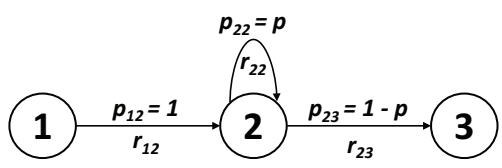

Figure 2. Example 2 of a Markov chain usage model. Arcs are annotated with transitional probabilities and arc reliabilities.

$$
\begin{aligned}
& \mathscr{F}_{1}=Q \otimes F_{1}=\left[\begin{array}{lll}
0 & \frac{1-r_{12}}{2} & \frac{1-r_{13}}{2} \\
0 & \frac{1-r_{22}}{2} & \frac{1-r_{23}}{2}
\end{array}\right] \quad U=\left[\begin{array}{l}
1 \\
1 \\
1
\end{array}\right] \\
& \left(I-\dot{\mathscr{R}}_{1}\right)^{-1} \mathscr{F}_{1}=\left[\begin{array}{ccc}
0 & \frac{1-r_{12}}{2}+\frac{\frac{r_{12}}{4}\left(1-r_{22}\right)}{1-\frac{r_{22}}{2}} & \frac{1-r_{13}}{2}+\frac{\frac{r_{12}}{4}\left(1-r_{23}\right)}{1-\frac{r_{22}}{2}} \\
0 & \frac{1-r_{22}}{2} & \frac{1-r_{23}}{2} \\
1-\frac{r_{22}}{2} & {\left[\begin{array}{c}
\frac{1-\frac{r_{22}}{2}-\frac{r_{13}}{2}+\frac{r_{13} r_{22}}{4}-\frac{r_{12} r_{23}}{4}}{1-\frac{r_{22}}{2}}
\end{array}\right]} \\
F^{*}=\left(I-\dot{\mathscr{R}}_{1}\right)^{-1} \mathscr{F}_{1} U=\left[\begin{array}{c}
1-\frac{r_{22}}{2}-\frac{r_{23}}{2} \\
1-\frac{r_{22}}{2}
\end{array}\right.
\end{array}\right] \\
& R^{*}=1-F^{*}=\left[\begin{array}{c}
\frac{\frac{r_{13}}{2}-\frac{r_{13} r_{22}}{4}+\frac{r_{12} r_{23}}{4}}{1-\frac{r_{22}}{2}} \\
\frac{\frac{r_{23}}{2}}{1-\frac{r_{22}}{2}}
\end{array}\right]
\end{aligned}
$$

By our new formula:

$$
\begin{aligned}
& W=\left[\begin{array}{c}
\frac{r_{13}}{2} \\
\frac{r_{23}}{2}
\end{array}\right] \quad R^{*}=\left(I-\dot{\mathscr{R}}_{1}\right)^{-1} W=\left[\begin{array}{c}
\frac{r_{13}}{2}+\frac{\frac{r_{12} r_{23}}{4}}{1-\frac{r_{22}}{2}} \\
\frac{r_{23}}{2} \\
1-\frac{r_{22}}{2}
\end{array}\right] \\
& =\left[\begin{array}{c}
\frac{\frac{r_{13}}{2}-\frac{r_{13} r_{22}}{4}+\frac{r_{12} r_{23}}{4}}{1-\frac{r_{22}}{2}} \\
\frac{r_{23}}{2} \\
1-\frac{r_{22}}{2}
\end{array}\right]
\end{aligned}
$$

We may also compute the single use reliability (SUR) directly based on its definition given the model structure. To compute the probability of a randomly chosen use (path) being successful, we compute the weighted sum of path reliabilities, with weights being the path probabilities.

By the definition of single use reliability:

$$
\begin{aligned}
& S U R=\sum_{i=0}^{\infty}\left(\frac{1}{2} \cdot\left(\frac{1}{2}\right)^{i} \cdot \frac{1}{2} \cdot r_{12} \cdot r_{22}^{i} \cdot r_{23}\right)+\frac{r_{13}}{2}=\frac{r_{12} r_{23}}{4} \sum_{i=0}^{\infty}\left(\frac{r_{22}}{2}\right)^{i}+ \\
& \frac{r_{13}}{2}=\frac{\frac{r_{12} r_{23}}{4}}{1-\frac{r_{22}}{2}}+\frac{r_{13}}{2}=\frac{\frac{r_{13}}{2}-\frac{r_{13} r_{22}}{4}+\frac{r_{12} r_{23}}{4}}{1-\frac{r_{22}}{2}}
\end{aligned}
$$

Figure 2 illustrates our second example of a Markov chain usage model.

By Stacy and Poore's formula:

$$
\begin{aligned}
& P=\left[\begin{array}{ccc}
0 & 1 & 0 \\
0 & p & 1-p \\
0 & 0 & 0
\end{array}\right] \quad Q=\left[\begin{array}{ccc}
0 & 1 & 0 \\
0 & p & 1-p
\end{array}\right] \quad \dot{Q}=\left[\begin{array}{ll}
0 & 1 \\
0 & p
\end{array}\right] \\
& R_{1}=\left[\begin{array}{lll}
r_{11} & r_{12} & r_{13} \\
r_{21} & r_{22} & r_{23}
\end{array}\right] \quad \dot{R}_{1}=\left[\begin{array}{ll}
r_{11} & r_{12} \\
r_{21} & r_{22}
\end{array}\right] \quad I=\left[\begin{array}{ll}
1 & 0 \\
0 & 1
\end{array}\right] \\
& \dot{\mathscr{R}}_{1}=\dot{Q} \otimes \dot{R}_{1}=\left[\begin{array}{cc}
0 & r_{12} \\
0 & r_{22} p
\end{array}\right] \quad I-\dot{\mathscr{R}}_{1}=\left[\begin{array}{cc}
1 & -r_{12} \\
0 & 1-r_{22} p
\end{array}\right]
\end{aligned}
$$




$$
\begin{aligned}
& \left(I-\dot{\mathscr{R}}_{1}\right)^{-1}=\frac{1}{1-r_{22} p}\left[\begin{array}{cc}
1-r_{22} p & r_{12} \\
0 & 1
\end{array}\right]=\left[\begin{array}{ll}
1 & \frac{r_{12}}{1-r_{22} p} \\
0 & \frac{1}{1-r_{22} p}
\end{array}\right] \\
& F_{1}=1-R_{1}=\left[\begin{array}{lll}
1-r_{11} & 1-r_{12} & 1-r_{13} \\
1-r_{21} & 1-r_{22} & 1-r_{23}
\end{array}\right] \\
& \mathscr{F}_{1}=Q \otimes F_{1}=\left[\begin{array}{ccc}
0 & 1-r_{12} & 0 \\
0 & p\left(1-r_{22}\right) & (1-p)\left(1-r_{23}\right)
\end{array}\right] \\
& \left(I-\dot{\mathscr{R}}_{1}\right)^{-1} \mathscr{F}_{1}=\left[\begin{array}{ccc}
0 & 1-r_{12}+\frac{p\left(1-r_{22}\right) r_{12}}{1-r_{22} p} & \frac{(1-p)\left(1-r_{23}\right) r_{12}}{1-r_{22} p} \\
0 & \frac{p\left(1-r_{22}\right)}{1-r_{22} p} & \frac{(1-p)\left(1-r_{23}\right)}{1-r_{22} p}
\end{array}\right] \\
& U=\left[\begin{array}{l}
1 \\
1 \\
1
\end{array}\right] \quad F^{*}=\left(I-\dot{\mathscr{R}}_{1}\right)^{-1} \mathscr{F}_{1} U=\left[\begin{array}{c}
\frac{1-r_{22} p-r_{12} r_{23}+r_{12} r_{23} p}{1-r_{22} p} \\
\frac{1-r_{22} p-r_{23}+r_{23} p}{1-r_{22} p}
\end{array}\right] \\
& R^{*}=1-F^{*}=\left[\begin{array}{c}
\frac{r_{12} r_{23}-r_{12} r_{23} p}{1-r_{22} p} \\
\frac{r_{23}-r_{23} p}{1-r_{22} p}
\end{array}\right]
\end{aligned}
$$

By our new formula:

$$
\begin{aligned}
& W=\left[\begin{array}{c}
0 \\
(1-p) r_{23}
\end{array}\right] \quad R^{*}=\left(I-\dot{\mathscr{R}}_{1}\right)^{-1} W= \\
& {\left[\begin{array}{ll}
1 & \frac{r_{12}}{1-r_{22} p} \\
0 & \frac{1}{1-r_{22} p}
\end{array}\right]\left[\begin{array}{c}
0 \\
(1-p) r_{23}
\end{array}\right]=\left[\begin{array}{c}
\frac{r_{12} r_{23}-r_{12} r_{23} p}{1-r_{22} p} \\
\frac{r_{23}-r_{23} p}{1-r_{22} p}
\end{array}\right]}
\end{aligned}
$$

By the definition of single use reliability:

$$
\begin{aligned}
& S U R=\sum_{i=0}^{\infty} 1 \cdot p^{i}(1-p) \cdot r_{12} \cdot r_{22}^{i} \cdot r_{23}=\sum_{i=0}^{\infty} r_{12} r_{23}(1-p)\left(p r_{22}\right)^{i}= \\
& \frac{r_{12} r_{23}(1-p)}{1-p r_{22}}=\frac{r_{12} r_{23}-r_{12} r_{23} p}{1-r_{22} p}
\end{aligned}
$$

\section{Conclusion and Future Work}

Statistical testing based on a Markov chain usage model has been well established in theory and proved sound and effective in practice $[6,5,10,8,11,14,16,15]$, with tools available to support all the stages of testing and to automate the testing process $[1,9]$. This paper presents a simpler way to compute the system end-to-end reliability mean, not through the system failure probability (or unreliability) but directly, following the arc-based Bayesian model [10, 12]. We illustrate it with two examples and compare the results with those obtained from the old analysis.

Work is under way to implement the new formula in the supporting tool JUMBL, and to examine the computation of the single use reliability variance to find an equivalent but simpler, more direct, and more intuitive derivation as well (the current one is complex and counter-intuitive).

\section{Acknowledgements}

This work was generously funded by Ontario Systems through the NSF Security and Software Engineering Research Center $\left(S^{2} E R C\right)$.

\section{References}

[1] 2017. J Usage Model Builder Library (JUMBL). Software Quality Research Laboratory, The University of Tennessee.
http://jumbl.sourceforge.net/jumblTop.html.

[2] T. Bauer, T. Beletski, F. Boehr, R. Eschbach, D. Landmann, and J. Poore. From requirements to statistical testing of embedded systems. In Proceedings of the 4th International Workshop on Software Engineering for Automotive Systems, pages 3-9, Minneapolis, MN, 2007.

[3] L. Bouwmeester, G. H. Broadfoot, and P. J. Hopcroft. Compliance test framework. In Proceedings of the 2nd Workshop on Model-Based Testing in Practice, pages 97-106, Enscede, The Netherlands, 2009.

[4] K. W. Miller, L. J. Morell, R. E. Noonan, S. K. Park, D. M. Nicol, B. W. Murrill, and J. M. Voas. Estimating the probability of failure when testing reveals no failures. IEEE Transactions on Software Engineering, 18(1):33-43, 1992.

[5] J. H. Poore. Theory-practice-tools for automated statistical testing. DoD Software Tech News: Model-Driven Development, 12(4):20-24, 2010.

[6] J. H. Poore, L. Lin, R. Eschbach, and T. Bauer. Automated statistical testing for embedded systems. In J. Zander, I. Schieferdecker, and P. J. Mosterman, editors, ModelBased Testing for Embedded Systems in the Series on Computational Analysis and Synthesis, and Design of Dynamic Systems. CRC Press-Taylor \& Francis, 2011.

[7] J. H. Poore, H. D. Mills, and D. Mutchler. Planning and certifying software system reliability. IEEE Software, 10(1):88-99, 1993.

[8] J. H. Poore and C. J. Trammell. Application of statistical science to testing and evaluating software intensive systems. In M. L. Cohen, D. L. Steffey, and J. E. Rolph, editors, Statistics, Testing, and Defense Acquisition: Background Papers. National Academies Press, 1999.

[9] S. J. Prowell. JUMBL: A tool for model-based statistical testing. In Proceedings of the 36th Annual Hawaii International Conference on System Sciences, page 337c, Big Island, HI, 2003.

[10] S. J. Prowell and J. H. Poore. Computing system reliability using Markov chain usage models. Journal of Systems and Software, 40(4):199-222, 2004.

[11] S. J. Prowell, C. J. Trammell, R. C. Linger, and J. H. Poore. Cleanroom Software Engineering: Technology and Process. Addison-Wesley, Reading, MA, 1999.

[12] K. Sayre and J. H. Poore. A reliability estimator for model based software testing. In Proceedings of the 13th International Symposium on Software Reliability Engineering, pages 53-63, Annapolis, MD, 2002.

[13] K. Sayre and J. H. Poore. Automated testing of generic computational science libraries. In Proceedings of the 40th Annual Hawaii International Conference on System Sciences, page 277c, Big Island, HI, 2007.

[14] G. H. Walton, J. H. Poore, and C. J. Trammell. Statistical testing of software based on a usage model. Software Practice \& Experience, 25(1):97-108, 1995.

[15] J. A. Whittaker and J. H. Poore. Markov analysis of software specifications. ACM Transactions on Software Engineering and Methodology, 2(1):93-106, 1993.

[16] J. A. Whittaker and M. G. Thomason. A Markov chain model for statistical software testing. IEEE Transactions on Software Engineering, 30(10):812-824, 1994. 